\title{
Hydroxychloroquine and lupus flare: a good drug, but we need to do better
}

\author{
Mary K Crow (1) ,' Kyriakos A Kirou ${ }^{2}$
}

Most rheumatologists are well aware of the 1991 landmark study from the Canadian Hydroxychloroquine Study Group that reported results from a prospective randomised, double-blind study in which hydroxychloroquine (HCQ) was either continued or discontinued in patients with systemic lupus erythematosus (SLE) that were clinically stable for at least 3 months, although with significant disease activity (mean SLE Disease Activity Index (SLEDAI) 7.9 and 8.7, respectively). ${ }^{1}$ The study was small, including 25 and 22 patients in the two groups, but the message was clear: over 6 months, a clinical flare (new or worse disease manifestations) occurred 2.5 times more frequently in the group that discontinued HCQ. Follow-up for three additional years demonstrated a $57 \%$ reduction in major flares (including cases of lupus nephritis and vasculitis) for those continuing HCQ, although this was not statistically significant due to the small number of study subjects. ${ }^{2}$ The rationale for the study reflected the concerns of patients and physicians regarding the potential toxicities that might be associated with long-term use of that agent. At least in part as a consequence of the Canadian study, HCQ, first approved for treatment of SLE by the US Food and Drug Administration in 1955, is now the foundational therapy for nearly all patients with SLE. HCQ is generally considered a safe and effective medication in SLE. ${ }^{3}$ Retinal damage is arguably the most significant toxicity of the drug and increases with cumulative exposure to HCQ. It is rare in the first 5 years of treatment $(\leq 1 \%)$ but increases substantially after 16-20 years $(8 \%-20 \%){ }^{4}$ Risk factors, besides cumulative dose, include high daily dose relative to body weight, reduced renal function, older age, high body mass index and use of tamoxifen. ${ }^{45}$ New recommendations have suggested a decrease of HCQ daily dosage to $\leq 5 \mathrm{mg} / \mathrm{kg}$ of actual body

\footnotetext{
${ }^{1}$ Mary Kirkland Center for Lupus Research, Hospital for Special Surgery, New York, New York, USA

'Department of Medicine, Hospital for Special Surgery, New York, New York, USA
}

Correspondence to Professor Mary K Crow, Mary Kirkland Center for Lupus Research, Hospital for Special Surgery, New York, New York, USA; crowm@hss.edu weight. ${ }^{5}$ Of note, measuring blood levels of HCQ may help detect non-adherence and may predict both efficacy and retinal toxicity. ${ }^{4-11}$ Assessing the benefits versus risks of continuing or stopping HCQ therapy remains an important priority for patients, and defining the features of those who might sustain low disease activity or remission after discontinuation of that drug continues to be an issue for effective management of lupus disease. ${ }^{3-11}$

Guidance regarding the relative benefits and risks of continuing HCQ therapy in patients with SLE is presented in a new report based on data from the Systemic Lupus International Collaborating Clinics (SLICC) inception cohort. ${ }^{12}$ The SLICC investigators, a multinational group from 33 clinical sites, studied 1460 patients initiating HCQ therapy from among 1711 patients with SLE prospectively enrolled in the cohort from 1999 to 2019. The primary outcome of the study was time to the first of the following events indicating a flare: need for augmented therapy (including HCQ, chloroquine, glucocorticoids, immunosuppressive drugs or biologics), increase of $\geq 4$ in SLEDAI$2000(2 \mathrm{~K})$ or hospitalisation for SLE. Treatment augmentation was the most frequent flare outcome measure in all groups, while hospitalisation rates were minimal. Flares of patients decreasing or discontinuing HCQ compared with those maintaining the initial dose (for an average of 1.7 years for the groups maintaining and discontinuing HCQ and 2.0 years for those decreasing HCQ) were retrospectively analysed, and factors independently associated with flare were identified. Importantly, this large, multisite, multiinvestigator study confirmed the general observation published in the original Canadian study, although with the HR for flare (1.56) in the discontinuation group compared with those who maintained HCQ somewhat less compelling than that reported in the 1991 study (table 1). As might be expected, the HR for flare in those reducing HCQ (1.20) was less than $\mathrm{HR}$ for flare in those discontinuing HCQ, perhaps suggesting that judicious tapering of the drug can successfully maintain a level of protection from flare. In all groups, use of glucocorticoids and immunosuppressive medications was associated with higher risks of flare. Asians (from South Korea) had a lower risk of flare if they reduced HCQ dose. Patients without a college or university education were significantly more likely to flare on discontinuation of HCQ, supporting the well-documented important contribution of socioeconomic factors to outcomes of patients with SLE. ${ }^{13-16}$ While a recent report suggested that patients with SLE aged $\geq 55$ years-old who are in a low disease activity state (SELENA-SLEDAI scores of $\leq 4$ ) may successfully discontinue HCQ without increased risk of disease flare ${ }^{17}$ the SLICC group did not differentiate risk of flare in patients above or below age 50 . The two studies also differed in that $36 \%-40 \%$ of SLICC patients had a SLEDAI-2K score of $\geq 4$ and thus higher disease activity. SLICC patients of all ages with low lupus disease activity state (defined as SLEDAI-2K score of $<4$ and prednisone dose of $\leq 7.5 \mathrm{mg}$ / day) or in remission (SLEDAI-2K score of 0 and no glucocorticoids or immunosuppressives in the last year) had lower flare rates as expected, but reduction or discontinuation of HCQ also increased their flare risk. Most notably, the flare rates for all four patient groups were $\geq 30$ flares/100 person-years. The glass half empty interpretation is that flare rates remain unacceptably high for all groups, even when HCQ is maintained or only reduced.

While these new data support the utility of HCQ in reducing risk of flare, the study has important limitations that call for continued analysis of this and other cohorts. ${ }^{12}$ The SLICC cohort study benefits from access to a large number of patients but did not provide the reasons for tapering or discontinuing therapy and did not differentiate mildmoderate from severe disease flares. The occurrence of clinical flares was ascertained only once per year, so uncertainties remain in the time to flare from the indicated time zero and whether mild flares might have been missed. Degree of drug adherence was not confirmed, and HCQ blood levels, a valuable measure of adherence, were not assessed. ${ }^{18-20}$

Nonetheless, the data support the value of HCQ in limiting flares that resulted in increased or (re)started HCQ, prednisone, immunosuppressive or biological agents, or increased SLEDAI-2K scores by $\geq 4$.

In addition to data indicating the benefits of HCQ therapy with regard to flare, knowledge of the mechanisms responsible for the beneficial and harmful effects of HCQ can inform shared decision making in patient management. The benefits of HCQ have been traditionally attributed 
Table 1 Incidence rates of first flare per 100 person-years for the HCQ reduction or discontinuation groups versus HCQ maintenance group with corresponding HRs for flare (in parenthesis) from analysis of the Systemic Lupus International Collaborating Clinics cohort ${ }^{12}$

\begin{tabular}{lllll}
\hline & All patients & Low activity & Remission & No low activity \\
\hline Reduce HCQ & 40.0 vs 31.9 (1.20) & 37.5 vs $27.8(1.32)$ & 26.2 vs 13.2 (2.14) & 43.9 vs 39.8 (1.04) \\
Discontinue HCQ & 41.3 vs 30.0 (1.56) & 35.5 vs 26.6 (1.62) & 24.7 vs 12.2 (2.77) & 53.6 vs 36.4 (1.6) \\
\hline
\end{tabular}

HCQ, hydroxychloroquine .

to its capacity to alkalinise intracellular lysosomes, limiting antigen-presenting cell function. ${ }^{21}$ As the contributions of endosomal toll-like receptors (TLRs) in driving production of type I interferon and B-cell differentiation have gained support as key components of lupus pathogenesis, HCQmediated alkalinisation of those endosomal TLRs (primarily TLR7, 8 and 9) is assumed to be an important contributor to efficacy in SLE. ${ }^{10112223}$ Recent investigation of the functions mediated by HCQ has extended understanding of its beneficial and harmful effects (figure 1). ${ }^{23-27}$

As weak bases, HCQ and chloroquine, derivatives of 4-aminoquinoline, accumulate in intracellular acidic endolysosomes and neutralise their $\mathrm{pH}$, potentially altering protein processing and antigen presentation on major histocompaticility complex (MHC) class II molecules and inhibiting TLR signalling and the resultant production of type I interferon, proinflammatory cytokines and differentiation of autoantibody-producing

B cells. ${ }^{232526}$ Beyond its effects on endosomal $\mathrm{pH}, \mathrm{HCQ}$ directly binds to nucleic acids, favouring binding to the guanosinecytosine-rich sequences in the major groove of DNA and thereby potentially blocking the interaction of DNA with TLR9. ${ }^{24} 27$ Similarly, HCQ can bind RNA, inhibiting activation of RNA-sensing TLR7 and TLR8. The observed inhibition of cytokine secretion by HCQ may be attributable to its Golgi alkalinisation, impairing protein secretion. ${ }^{25}$

The potential for patients treated with HCQ to experience toxicity from that drug is primarily a function of daily dose, reflected in blood levels, and duration of treatment. ${ }^{25}$ For patients treated with HCQ for less than 5 years, it would appear that the mechanisms that abrogate production or secretion of type I interferon and other cytokines and are purported to limit antigen presentation are likely to outweigh the mechanisms that contribute to the toxicity of HCQ, most notably those that may impact vision. The risk of retinal

\section{Hydroxychloroquine: Mechanisms of Action}

Detrimental

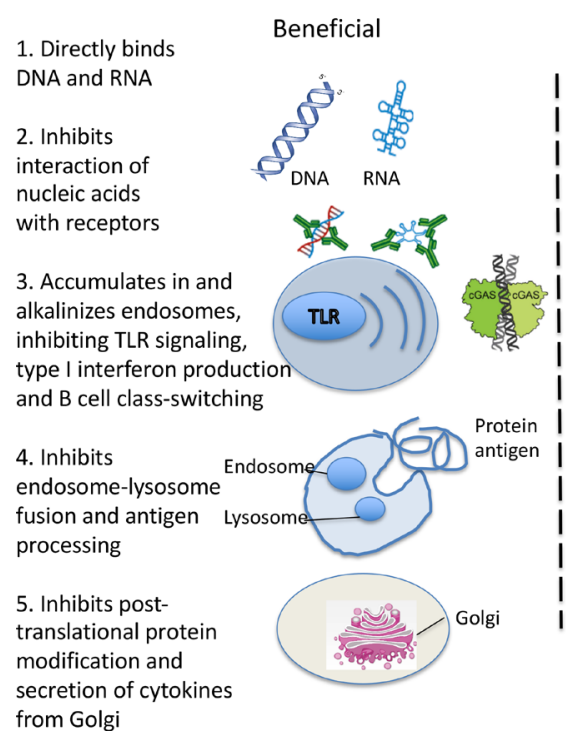

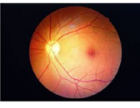

Retinopathy

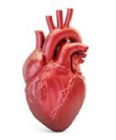

Cardiomyopathy

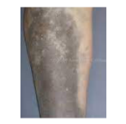

Skin hyperpigmentation
1. Inhibits autophagy, leading to accumulation of intracellular debris and lipofuscin

2. Inactivates lysosomal enzymes

3. Inhibits phagocyte function

4. Binds melanin

Figure 1 Mechanisms of HCQ contributing to beneficial and detrimental effects. While additional research is required to fully elucidate the relevant mechanisms of HCQ, many of the proposed mechanisms, including direct binding to nucleic acids, alkalinisation of endosomal compartments, inhibition of endosome-lysosome fusion and Golgi alkalinisation, resulting in impaired secretion of proinflammatory cytokines, impact pathogenic mechanisms operative in systemic lupus erythematosus. Inhibition of autophagy, a cellular process responsible for degrading spent cellular components, can result in accumulation of intracellular and extracellular debris, leading to deposition of lipofuscin and damage to cells. In addition, HCQ binds to melanin, contributing to changes in skin pigmentation. CGAS, cyclic GMP-AMP synthase; HCQ, hydroxychloroquine; TLR, toll-like receptor. toxicity is estimated to be $<2 \%$ in the first 10 years in patients taking HCQ of $5 \mathrm{mg} /$ $\mathrm{kg}$ of their actual weight. ${ }^{525}$ Many of the toxicities attributable to HCQ may involve its inhibition of autophagy. ${ }^{28}$ Efficient autophagy contributes to degradation and clearance of cell organelles, and impaired autophagy can result in accumulation of damaging intracellular and extracellular aggregates. HCQ may initiate retinal damage by binding to melanin in retinal epithelium, inducing intracellular accumulation of lipofuscin (lipid-containing material derived from lysosomes) by inhibiting autophagy, followed by damage to photoreceptors. ${ }^{25} 2829$ Cardiac toxicity leading to conduction abnormalities and toxic myopathy may also occur and, like the ocular manifestations, appears to be dose related. In cardiac myocytes, HCQ's capacity to alkalinise lysosomal contents can inhibit enzyme function and can result in accumulation of phospholipids that are not properly degraded, with generation of lipid bodies. ${ }^{25} 30$ Skin pigmentation at sites of bruising may be a result of HCQ promoting accumulation of cell debris, including melanin, followed by stimulation of melanogenesis.

As is the case for any discussion of disease management with patients, knowledge of the benefits of HCQ as well as potential for harm should be based on the most reliable data available. The study of the SLICC cohort confirms the impact of HCQ therapy on reducing risk of lupus flare and provides data that can inform discussions between patients and physicians. Knowledge of the drug's molecular mechanisms, particularly those consistent with current concepts of lupus pathogenesis, supports the case for inclusion of HCQ in therapeutic regimens. There is clear benefit in the setting of relatively modest risks, but those risks increase with duration of therapy. The message to patients will remain nuanced pending future research that might define biological predictors of flare and for patients desiring tapering or discontinuation of HCQ. As the HRs for patients decreasing HCQ in the SLICC study were lower than for those discontinuing the drug, perhaps judicious tapering of HCQ, guided by monitoring of blood levels, might optimise 
the flare reduction benefits of HCQ while minimising risk of dose-related toxicities. Addressing barriers to care attributable to socioeconomic circumstances and limited educational opportunities might also improve compliance, reduce likelihood of flare and improve disease outcomes.

Perhaps the most striking and instructive message from the SLICC cohort study is that even in the context of continued HCQ therapy, flares are unacceptably high. The lupus research community has its marching orders, pointing to the need for improved understanding of underlying pathogenic mechanisms, therapeutic target identification, and development of effective and safe therapeutics that might ultimately surpass the benefits of HCQ.

\section{Handling editor Josef S Smolen}

Contributors Both authors reviewed the relevant accepted manuscript (Bernatsky et al) and contributed to writing and editing the submitted editorial.

Funding The authors have not declared a specific grant for this research from any funding agency in the public, commercial or not-for-profit sectors.

Competing interests None declared.

Patient and public involvement Patients and/or the public were not involved in the design, conduct, reporting or dissemination plans of this research.

Patient consent for publication Not applicable.

Ethics approval This study does not involve human participants.

Provenance and peer review Commissioned; externally peer reviewed.

(C) Author(s) (or their employer(s)) 2022. No commercial re-use. See rights and permissions. Published by BMJ.

\section{A Check for updates}

To cite Crow MK, Kirou KA. Ann Rheum Dis 2022;81:303-305.

Received 29 November 2021

Accepted 6 December 2021

Published Online First 17 January 2022

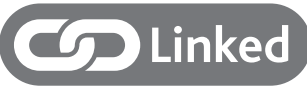

http://dx.doi.org/10.1136/annrheumdis-2021221295

Ann Rheum Dis 2022;81:303-305.

doi:10.1136/annrheumdis-2021-221590
ORCID iD

Mary K Crow http://orcid.org/0000-0002-7881-2020

\section{REFERENCES}

1 Canadian Hydroxychloroquine Study Group. A randomized study of the effect of withdrawing hydroxychloroquine sulfate in systemic lupus erythematosus. N Engl J Med 1991;324:150-4.

2 Tsakonas E, Joseph L, Esdaile JM, et al. A longterm study of hydroxychloroquine withdrawal on exacerbations in systemic lupus erythematosus. The Canadian hydroxychloroquine Study Group. Lupus 1998;7:80-5.

3 Ruiz-Irastorza G, Ramos-Casals M, Brito-Zeron P, et al. Clinical efficacy and side effects of antimalarials in systemic lupus erythematosus: a systematic review. Ann Rheum Dis 2010;69:20-8.

4 Petri M, Elkhalifa M, Li J, et al. Hydroxychloroquine blood levels predict hydroxychloroquine retinopathy. Arthritis Rheumatol 2020;72:448-53.

5 Rosenbaum JT, Costenbader KH, Desmarais J, et al. American College of rheumatology, American Academy of dermatology, rheumatologic dermatology Society, and American Academy of ophthalmology 2020 joint statement on hydroxychloroquine use with respect to retinal toxicity. Arthritis Rheumatol 2021;73:908-11.

6 Costedoat-Chalumeau N, Galicier L, Aumaître O, et al. Hydroxychloroquine in systemic lupus erythematosus: results of a French multicentre controlled trial (plus study). Ann Rheum Dis 2013;72:1786-92.

7 Mok CC, Penn HJ, Chan KL, et al. Hydroxychloroquine serum concentrations and flares of systemic lupus erythematosus: a longitudinal cohort analysis. Arthritis Care Res 2016:68:1295-302.

8 Geraldino-Pardilla L, Perel-Winkler A, Miceli J, et al. Association between hydroxychloroquine levels and disease activity in a predominantly Hispanic systemic lupus erythematosus cohort. Lupus 2019:28:862-7.

9 Garg S, Unnithan R, Hansen KE, et al. Clinical Significance of Monitoring Hydroxychloroquine Levels in Patients With Systemic Lupus Erythematosus: A Systematic Review and Meta-Analysis. Arthritis Care Res 2021;73:707-16.

10 Nirk EL, Reggiori F, Mauthe M. Hydroxychloroquine in rheumatic autoimmune disorders and beyond. EMBO Mol Med 2020;12:e12476.

11 Schrezenmeier E, Dörner T. Mechanisms of action of hydroxychloroquine and chloroquine: implications for rheumatology. Nat Rev Rheumatol 2020;16:155-66.

12 Almeida-Brasil CC, Hanly JG, Urowitz M, et al. Flares after hydroxychloroquine reduction or discontinuation: results from the systemic lupus international collaborating clinics (SLICC) inception cohort. Ann Rheum Dis 2022;81:369-77.

13 Fernández M, Alarcón GS, Calvo-Alén J, et al. A multiethnic, multicenter cohort of patients with systemic lupus erythematosus (SLE) as a model for the study of ethnic disparities in SLE. Arthritis Rheum 2007; 57:576-84.

14 Sun K, Eudy AM, Criscione-Schreiber LG, et al. Racial disparities in medication adherence between African American and Caucasian patients with systemic lupus erythematosus and their associated factors. ACR Open Rheumatol 2020:2:430-7.

15 DeQuattro K, Yelin E, status S. Socioeconomic status, health care, and outcomes in systemic lupus erythematosus. Rheum Dis Clin North Am 2020:46:639-49.

16 Pryor KP, Barbhaiya M, Costenbader KH, et al. Disparities in lupus and lupus nephritis care and outcomes among US Medicaid beneficiaries. Rheum Dis Clin North Am 2021;47:41-53.

17 Fernandez-Ruiz R, Bornkamp N, Kim MY, et al. Discontinuation of hydroxychloroquine in older patients with systemic lupus erythematosus: a multicenter retrospective study. Arthritis Res Ther 2020;22:191.

18 Costedoat-Chalumeau N, Amoura Z, Hulot J-S, et al. Low blood concentration of hydroxychloroquine is a marker for and predictor of disease exacerbations in patients with systemic lupus erythematosus. Arthritis Rheum 2006;54:3284-90.

19 Yeon Lee J, Lee J, Ki Kwok S, et al. Factors related to blood hydroxychloroquine concentration in patients with systemic lupus erythematosus. Arthritis Care Res 2017;69:536-42.

20 Blanchet B, Jallouli M, Allard M, et al. Hydroxychloroquine levels in patients with systemic lupus erythematosus: whole blood is preferable but serum levels also detect non-adherence. Arthritis Res Ther 2020;22:223.

21 Fox Rl, Kang H-I. Mechanism of action of antimalarial drugs: inhibition of antigen processing and presentation. Lupus 1993:2:9-12.

22 Kuznik A, Bencina M, Svajger U, et al. Mechanism of endosomal TLR inhibition by antimalarial drugs and imidazoquinolines. J Immunol 2011;186:4794-804.

23 Richard SA, Kampo S, Hechavarria ME, et al. Elucidating the pivotal immunomodulatory and anti-inflammatory potentials of chloroquine and hydroxychloroquine. J Immunol Res 2020;2020:1-13.

24 An J, Minie M, Sasaki T, et al. Antimalarial drugs as immune modulators: new mechanisms for old drugs. Annu Rev Med 2017:68:317-30.

25 Muller R. Systemic toxicity of chloroquine and hydroxychloroquine: prevalence, mechanisms, risk factors, prognostic and screening possibilities. Rheumatol Int 2021:41:1189-202.

26 Torigoe M, Sakata K, Ishii A, et al. Hydroxychloroquine efficiently suppresses inflammatory responses of human class-switched memory B cells via toll-like receptor 9 inhibition. Clin Immunol 2018;195:1-7.

27 Sarkar S, Roy S, Singh PC. Groove switching of hydroxychloroquine modulates the efficacy of binding and induced stability to DNA. J Phys Chem B 2021;125:6889-96.

28 Münz C. Autophagy beyond intracellular MHC class II antigen presentation. Trends Immunol 2016:37:755-63.

29 Jorge A, Ung C, Young LH, et al. Hydroxychloroquine retinopathy - implications of research advances for rheumatology care. Nat Rev Rheumatol 2018;14:693-703.

30 Yogasundaram H, Hung W, Paterson ID, et al. Chloroquine-induced cardiomyopathy: a reversible cause of heart failure. ESC Heart Fail 2018;5:372-5. 